\title{
Sistemas de Informações Geográficas e seus visualizadores tridimensionais para o ensino do projeto de Arquitetura e Urbanismo
}

\author{
Renato César Ferreira de Souza*
}

Resumo Estuda-se o apoio de Sistemas de Informações Geográficas (SIG) para o ensino de projetos de Arquitetura e Urbanismo. Esperava-se que estudantes alcançassem maior rapidez na tomada de decisões gerando hipóteses projetuais e testando-as, o quê hipoteticamente, resultaria em soluções de projeto mais criativas e detalhadas. Observação e avaliação dos produtos gerados demonstraram que o potencial das ferramentas depende do ajuste do tempo de aprendizagem do uso do SIG. Os resultados apresentaram projetos majoritariamente derivados de hipóteses únicas. Pesquisas futuras estudarão a criatividade na geração de hipóteses projetuais com apoio do instrumental, mas considerando diversos problemas de projeto na proposta de ensino.

Palavras-chave: SIG, visualizadores de SIG, projeto arquitetônico e urbano, educação superior.

\section{Geographic Information Systems and their 3D viewers for the teaching on projects for Ar- chitecture and Urban Design}

\begin{abstract}
This article studies the support of Geographic Information Systems (GIS) for the teaching of Architecture and Urbanism projects. Students were expected to achieve faster decision making by generating design hypotheses and testing them, what hypothetically would result in more creative and detailed design solutions. Observation and evaluation of the generated products showed that the potential of these tools depends on the adjustment of learning time of GIS. Mostly of the resulting projects presented derived from an unique hypotheses. Future research will study creativity in the generation of design hypotheses with the support of those instruments, considering hypothesizing several design solutions for problems in the teaching proposal.
\end{abstract}

Key words: GIS, GIS viewers, architectural and urban design, higher education.

\section{Sistemas de Información Geográfica y sus visualizadores tridimensionales para la en- señanza del proyecto de Arquitectura y Urbanismo}

Resumen Se estudia el apoyo de Sistemas de Información Geográfica (SIG) para la enseñanza de proyectos de Arquitectura y Urbanismo. Se esperaba que los estudiantes alcanzara mayor rapidez en la toma de decisiones generando hipótesis de diseño y probándolas, lo que, hipotéticamente, resultaría en soluciones de diseño más creativas y detalladas. La observación y evaluación de los productos generados han demostrado que el potencial de las herramientas depende del ajuste del tiempo de aprendizaje del uso del SIG. Los resultados presentaron proyectos mayoritariamente derivados de hipótesis únicas. Las investigaciones futuras estudiarán la creatividad en la generación de hipótesis proyectuales con apoyo del instrumental, pero considerando diversos problemas de proyecto en la propuesta de enseñanza.

Palavras clave: SIG, visualizadores de SIG, proyecto arquitectónico y urbano, educación superior. 


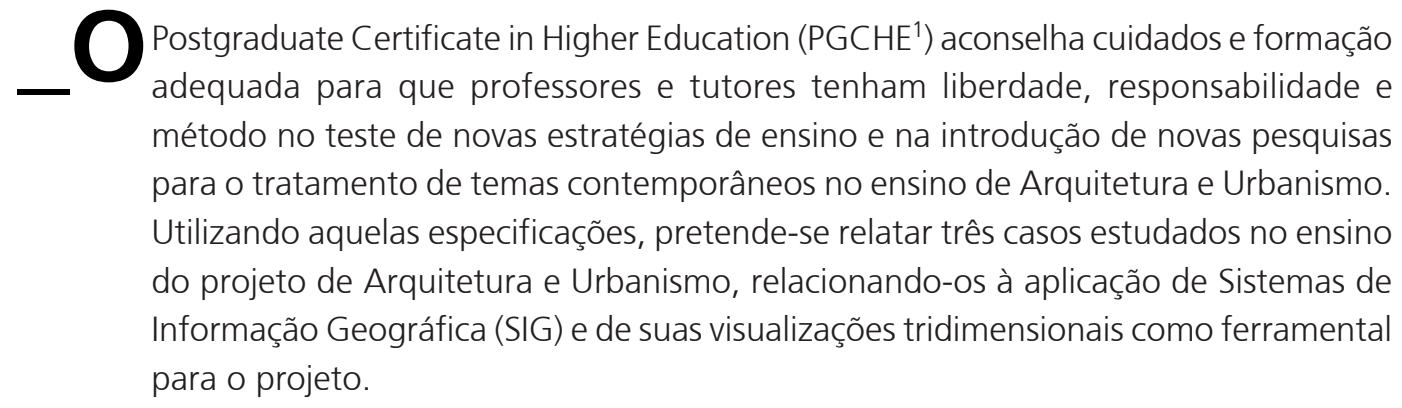

\section{O problema}

A introdução do uso dos SIG's no ensino de projeto em Arquitetura e Urbanismo especificamente em temas limítrofes entre espaços públicos e privados - é problemática: se por um lado aquelas ferramentas já se utilizam no ensino da Geografia, Engenharias, Negócios, Comunicações, dentre outras áreas, no ensino de projeto arquitetônico e urbano elas têm avançado timidamente (MONSUR 2014). Em todos os outros campos, os SIG's têm efetivamente contribuído nas soluções de problemas sociais, econômicos, culturais, etc.. Mas para a Arquitetura e o Urbanismo, tal potencial merece ainda pesquisas endereçadas aos novos métodos de ensino e aprendizagem, e seu potencial. A evolução dos SIG's pode ser explicada pelas condições históricas do desenvolvimento da Geografia, nas últimas três décadas (MCCULLOUGH, 2004). Diferentemente da Arquitetura e Urbanismo, que desmaterializou seu objeto de estudo - o lugar - devido à crença generalizada na ilusão de que a Tecnologia da Informação (TI) determinaria inexoravelmente uma revolução social com a extinção das distâncias e limites territoriais (ARNOLD, 2003; CONRAD, 2006; GRAHAN, 1998; GREENFIELD; SHEPARD, 2007; WINOGRAD; FLORES; FERNANDO, 1988), a Geografia, ao contrário, avançou, desde a década de 1960 no estudo sobre a localidade (MCCULLOUGH, 2004) conjugando o seu conhecimento com os recursos desenvolvidos pela TI. Passados esses anos, ela conseguiu se abrir à transdiciplinariedade, sem perder o foco de seus

* Renato César Ferreira de Souza é Arquiteto e Urbanista, professor associado do curso de Arquitetura e Urbanismo da EAUFMG, professor credenciado permanente do Núcleo de Pós-graduação da EAUFMG, professor colaborador do Núcleo de Pós-graduação da Faculdade de Medicina da UFMG. ORCID: <https://orcid.org/00000002-1954-9496>.

1 "Postgraduate Certificate in Higher Education - Wikipedia." $<$ https://en.wikipedia.org/wiki/ Postgraduate_Certificate_in_Higher_Education>. Acessado em 29 abr. 2017. objetivos ao se conjugar com os estudos econômicos, políticos e socais, dentre outros. Contemporaneamente, estamos presenciando uma explosão no uso de mapeamentos, elaborados com operações básicas dos SIG's ou simplesmente tomados de fontes secundárias, para apoiar tímidas análises e fundamentações do projeto nos trabalhos estudantis de Arquitetura e Urbanismo. Isso indica, ao menos, um crescente interesse pela ferramenta aplicada ao projeto. Igualmente, o aparecimento de laboratórios de ensino de geoprocessamento em alguns cursos de Arquitetura e Urbanismo, juntamente com o surgimento do Geodesign (JORGENSEN, 2012) têm corroborado com mais motivos para se pesquisar em que medida e de que modos disciplinas de projeto podem incorporar o conhecimento dos sistemas de informação geográficas e como estes efetivamente podem qualificar os resultados (MONSUR, 2014). Tentando contribuir nesse sentido, este artigo busca relatar criticamente três estudos de caso sobre o uso de SIG aplicado em disciplina de projeto para o curso de Arquitetura da Universidade Federal de Minas Gerais (UFMG), entre os anos de 2014 a 2016. 
2 C.f.: Métodos de Análise Multicritérios in DE ALMEIDA et al (2015).

\section{Hipóteses}

O uso dos SIG's pode contribuir para a aprendizagem do projeto urbano e arquitetônico na medida em que amplia o universo de conhecimentos nos quais se inserem os problemas projetuais. Permite uma rápida visualização de dados complexos pelos estudantes e acelera a tomada de decisões. Permite que as análises iniciais do problema projetual sejam abrangentes, considerando ao mesmo tempo diversos critérios ${ }^{2}$ no estudo das situações complexas. Permite que sejam analisados possíveis efeitos de diferentes agentes envolvidos em cenários prospectivos. Todas estas afirmações anteriores são as hipóteses sobre as habilidades que os estudantes adquiririam na disciplina e foram testadas com o estudo de casos apresentado a seguir.

\section{Objetos de estudo}

Os casos constituem-se na análise de trabalhos e processos individuais de estudantes em três bimestres da disciplina "Espaços Públicos e Privados nas Perspectivas Contemporâneas", integrante do curso de Arquitetura da UFMG. A disciplina possui 60 horas/aulas bimestrais e não solicita pré-requisições aos estudantes que já tenham cursado os módulos de fundamentação. Os estudantes matriculados variam do $4^{\circ}$ ao $9^{\circ}$ período, sendo isso uma estratégia pedagógica, na tentativa de criar um ambiente de troca e compartilhamento de experiências entre estudantes iniciantes e veteranos.

\section{Objetivos educacionais}

Após cursar a disciplina, o estudante deveria ser estar apto a:

Elaborar anteprojeto de desenho urbano básico em área já ocupada na cidade de Belo Horizonte, utilizando a inserção de projetos básicos de arquitetura e considerando os aspectos sociais, a morfologia, a infraestrutura e a ambiência urbana;

Analisar e interpretar os contextos locais e as análises em sala, elaborando a justificação das propostas de acordo com eles;

Elaborar proposições de regulamentos específicos para soluções particularizadas, de acordo com o contexto local mais do que através da padronização dos usos e ocupações do solo;

Utilizar racionalmente novas metodologias de projeto, juntamente com novas tecnologias, na proposição de soluções originais;

Representar o desenho arquitetônico e urbano satisfatoriamente, de modo a esclarecer todos os elementos da proposta a partir de desenhos técnicos e visualizações de modelos tridimensionais.

\section{O terreno}

A área trabalhada compreendeu os terrenos indicados na figura 1, e o trabalho foi um exercício de desenho urbano com a inserção de edificações residenciais, comerciais e institucionais cujos projetos deveriam ser adaptados observando o padrão legal 


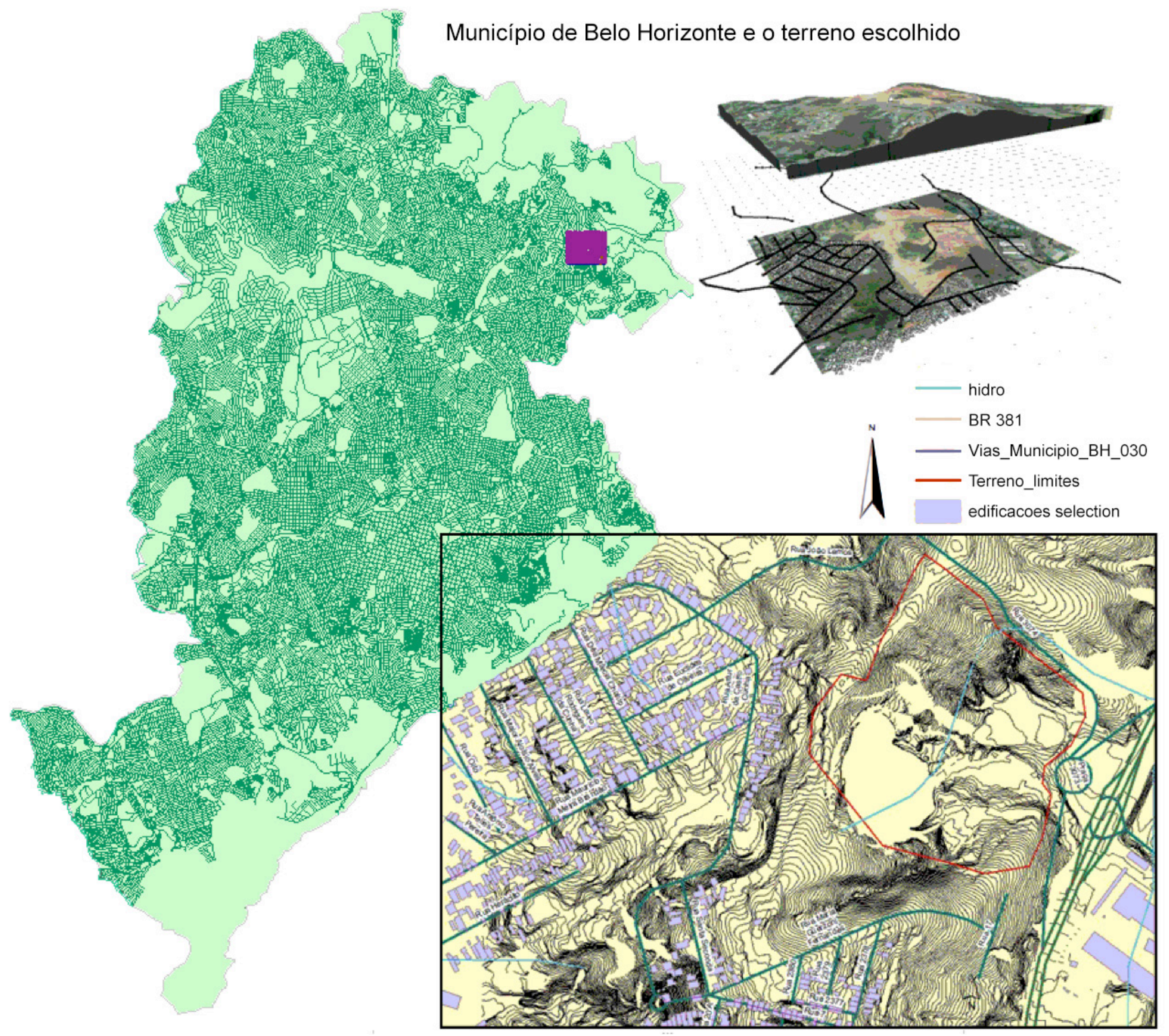

Figura 1: O terreno, em Minas Gerais, Brasil. Fonte: O autor, 2015.

3 "Minha Casa Minha Vida - Habitação Popular I Caixa." <http:// www.caixa.gov.br/voce/habitacao/minha-casa-minha-vida/Paginas/default.aspx>. Acessado em 30 abril 2017. gerado na criação do empreendimento, juntamente com as bases técnicas advindas do debate sobre os problemas habitacionais da circunvizinhança $(\mathrm{MCMV})^{3}$.

O terreno encontra-se em área sujeita a critérios urbanísticos especiais, por conter uma cava desativada, resultante da extração de hematita. Deve ter baixa densidade demográfica e uma maior taxa de permeabilização, em vista do interesse público na proteção ambiental.

Três desafios moveram as rodadas de desenho: reduzir os problemas de habitabilidade do conjunto, sobretudo no estudo do seu assentamento, de modo a garantir visibilidade, vigilância e segurança sem necessidade da criação de condomínios; possibilitar futuras alternativas para a acessibilidade, estudando o sistema de acesso à região; proporcionar 
conjuntos construtivos afirmativos, no sentido de integrá-los ao conjunto das ocupações circunvizinhas, buscando a redução de migrações voluntárias e a segregação social.

\section{Recursos utilizados}

Os estudantes possuíam seus próprios microcomputadores, com diferentes configurações, e com eles participavam das aulas. O professor lecionou 15 módulos de 4 horas/aula semanais nos 3 bimestres iniciais de 2014 a 2016, sempre utilizando um computador e um retroprojetor para explicação do instrumental e para análises coletivas. Adotaramse basicamente os seguintes programas: Depthmap ( ) (TURNER, 2013); Arcgis () (módulo Arcmap); e Cityengine ( $($ ESRI, 2016). Eles foram introduzidos na medida em que os conteúdos foram apresentados. Não houve treinamento específico para o uso deles, mas apenas a instrução dos comandos básicos de cada um, para o alcance dos objetivos educacionais. Dois bolsistas, um de Iniciação científica e outro de doutorado, já treinados no uso de software, tiravam dúvidas e complementavam o treinamento dos estudantes, de acordo com as necessidades individuais.

Os principais conceitos da Análise Configuracional (HILLIER; HANSON, 1984) ocuparam 4 horas/aula e o professor orientador da disciplina exemplificou o estudo da rede viária local, calculando em sala de aula, através do software Depthmap @ , as medidas de conectividade, integração e intermediação (betweeness) para as vias existentes nos arredores do terreno. A seguir, cada estudante gerou propostas de novas vias a serem implantadas, verificando, com o mesmo software, o impacto que a nova configuração causaria na região em termos da análise da segregação e integração de vias no conjunto e nos arredores (figura 2). Na discussão conceitual dos projetos propostos individualmente, as vias apontadas como segregadas pelo software eram tomadas para a localização de usos residencial e institucional de bairro (residências, escolas e Unidades Básica de Saúde), e as integradas para o uso comercial, institucional e praças públicas. As vias com maiores índices de intermediação (betweeness) foram consideradas apropriadas para localização de pontos de ônibus, serviços e comércios básicos, agência de correios, postos policiais, padarias, mercados e associação comunitária, por serem as vias de maior cruzamento do maior número de menores percursos entre pares de vias de todo conjunto. O zoneamento de cada trabalho foi justificado pelos estudos de conectividade juntamente com o estudo das soluções técnicas deduzidas a partir da sobreposição das propostas feitas ao mapa clinográfico do terreno, e de camadas com dados como demografia, renda, educação, saúde, segurança pública, todos eles espacializados através do software Arcgis@ , em demonstração pelo professor, em sala de aula, com dados censitários IBGE 2010.

4 "IBGE :: Instituto Brasileiro de Geografia e ...." <http://www. ibge.gov.br/>. Acessado em 30 abr. 2017.

\section{Psicomotricidade}

No sentido de compartilhar este estudo, é necessário enunciar os objetivos educacionais que visam introduzir alterações no comportamento dos estudantes, no sentido de capacitá-los a operar com destreza instrumentos necessários à profissão (ANDERSON et al., 2001).

Um médico cirurgião que não seja adestrado para usar bisturi pode ser uma ameaça para seus pacientes, por exemplo. No caso em estudo, os objetivos educacionais da área de psicomotricidade centraram-se na habilidade em usar o Cityengine (c) (ESRI, 
5 "CityEngine - Wikipedia." <https://en.wikipedia.org/wiki/ CityEngine>. Acessado em 30 abr. 2017

6 Computer Generated Architecture, traduzido aqui como "arquitetura gerada por computador".

Figura 2: Calculos de integração e intermediação para o município (a) e Conectividade local existente (b), e uma proposição(c). Na proposição o impacto das vias criadas resulta numa via muito integrada no interior do bairro (em vermelho), o que sugere a possibilidade de que ela se transforme em uma barreira, dividindo o território do projeto em duas comunidades separadas pela uma via e pelos declives mostrados na figura 1. Fonte: O autor, 2015. 2015a). Este software, desenvolvido desde $2008^{5}$ pela empresa Procedural Inc., em Zurique, difundiu-se após ser redesenhado e lançado pela ESRI@, em 2011. Em 2017 encontra-se na sua segunda versão. Trata-se de um visualizador tridimensional de SIG's, permitindo também a modelagem procedural de ambientes urbanos, criando grandes maquetes eletrônicas de cidades, com detalhamento apurado, permitindo vários tipos de simulações e estudos - desde a morfologia urbana até ao volume de tráfego suportado pelas vias, o uso e ocupação do solo urbano e outros. Modelos de edificações importados de outros modeladores preservam seus detalhes, podendo ser implantados na maquete eletrônica criada. O Cityengine $\odot$ tem total interoperabilidade com o Arcgis $\odot$, Autocad $\odot$ e SketchUp@ e permite adaptações, uma vez que é escrito na linguagem denominada $\mathrm{CGA}^{6}$, na qual as variáveis dos procedimentos (rules) podem ser escritas, adaptadas e visualizadas graficamente em painéis na interface gráfica. Por essa condição, os procedimentos podem ser modificados e podem contabilizar outros quantitativos, como preço do solo criado, quantidade de asfalto, posteamentos, mobiliários urbanos, tipos e quantidades de árvores e até o volume de tintas para a sinalização em arruamentos.

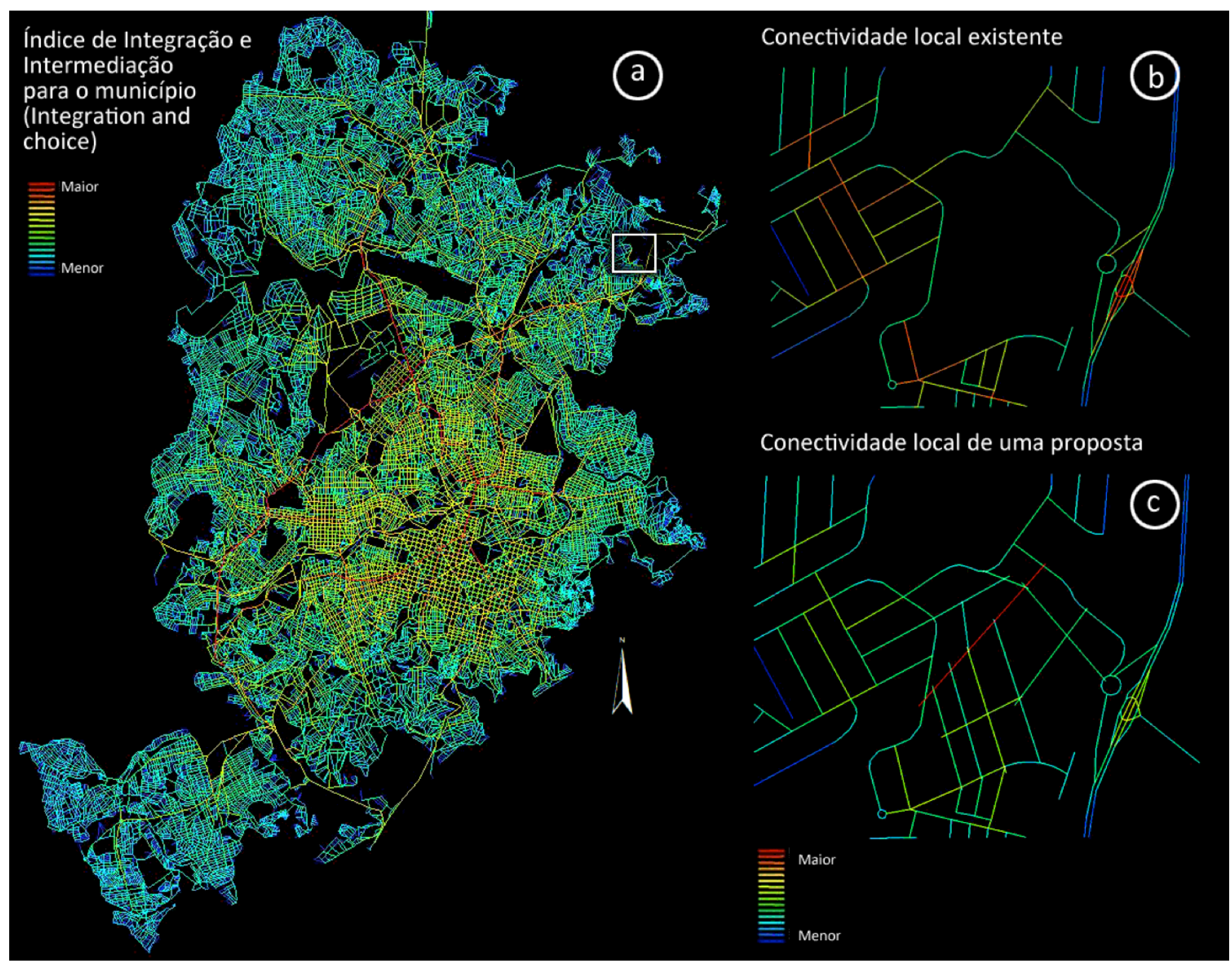


7 City Modelling Information, termo cunhado em referência ao BIM para projetos de Arquitetura e Engenharia.

8 Estudos globais e pontuais dos aspectos visíveis da cena urbana.
Enfim, trata-se de um software que se desenvolve na direção de modelar a informação da cidade, num primeiro esboço de sistema $\mathrm{CIM}^{7}$.

A figura 3 visa esclarecer as habilidades que se esperavam desenvolver nos estudantes, com o uso do Cityengine $\odot$, compreendidas nas seguintes etapas:

Desenhar os arruamentos, definir tamanhos de lotes e quadras, e especificar o envelope das edificações de acordo com a taxa de ocupação, coeficiente de aproveitamento, afastamentos e alturas máximas, compondo assim uma referência para o zoneamento e a legislação;

Decidir, quando fosse o caso, pelas divisões recursivas dos lotes e respectiva criação de áreas interiorizadas e verdes para pedestres no interior das quadras;

Ambientar as áreas de circulação de pedestres e atribuir um gradiente de privacidade;

Compor o recinto das ruas baseando-se em princípios do desenho urbano, decidindo sobre afastamentos e outros aspectos que garantissem a variedade e significado da imagem urbana. Indicar o uso de edificações específicas para residências, comércios, serviços, indústrias e edifícios comunitários, alocando espaço para praças contíguas às ruas;

Estudar a inserção e adaptação dos projetos arquitetônicos para edificações específicas, utilizando o envelope resultante da regulagem do uso e ocupação do solo, de forma a utilizar o número de pavimentos, afastamentos, área edificada, áreas permeáveis, compatibilizando pelo menos três projetos arquitetônicos;

Estudar a imagem urbana criada, incluindo elementos caracterizadores da cena urbana, estudos de visibilidade e integração (Isovistas e Viewsheds ${ }^{8}$ ), levando em consideração o terreno, prospecções de mudanças futuras das edificações e zoneamentos e edifícios de importância histórica;

Estudar a composição urbana considerando a simulação de densidade populacional e o volume de tráfego estimado, de acordo com dedução das densidades estimadas através do censo.

\section{Resultados}

Um total de 60 trabalhos de estudantes, constituídos de imagens e textos memoriais foram recebidos, analisados e avaliados ao final da disciplina. Anotações sistemáticas sobre o registro do processo individual, feitas pelo professor e assistentes foram também analisadas. Amostras das imagens geradas pelos estudantes são apresentadas na figura 4 .

Nela, a, b e c são visões da prospecção final da implantação. A morfologia urbana varia conforme as diferenças territoriais propostas. Em d, um estudo geral do uso e ocupação do solo proposto utiliza implantações genéricas. A adoção de uma área de proteção lindeira à rodovia e a preservação da área da cava minerada podem ser vistas em d, e, e $f$, e foram soluções que ocorreram em todos projetos. Vistas superiores foram representadas sem detalhes no Cityengine $\odot$ dado que ele permitia a simultaneidade de visualização de projeções ortogonais e de perspectivas. 


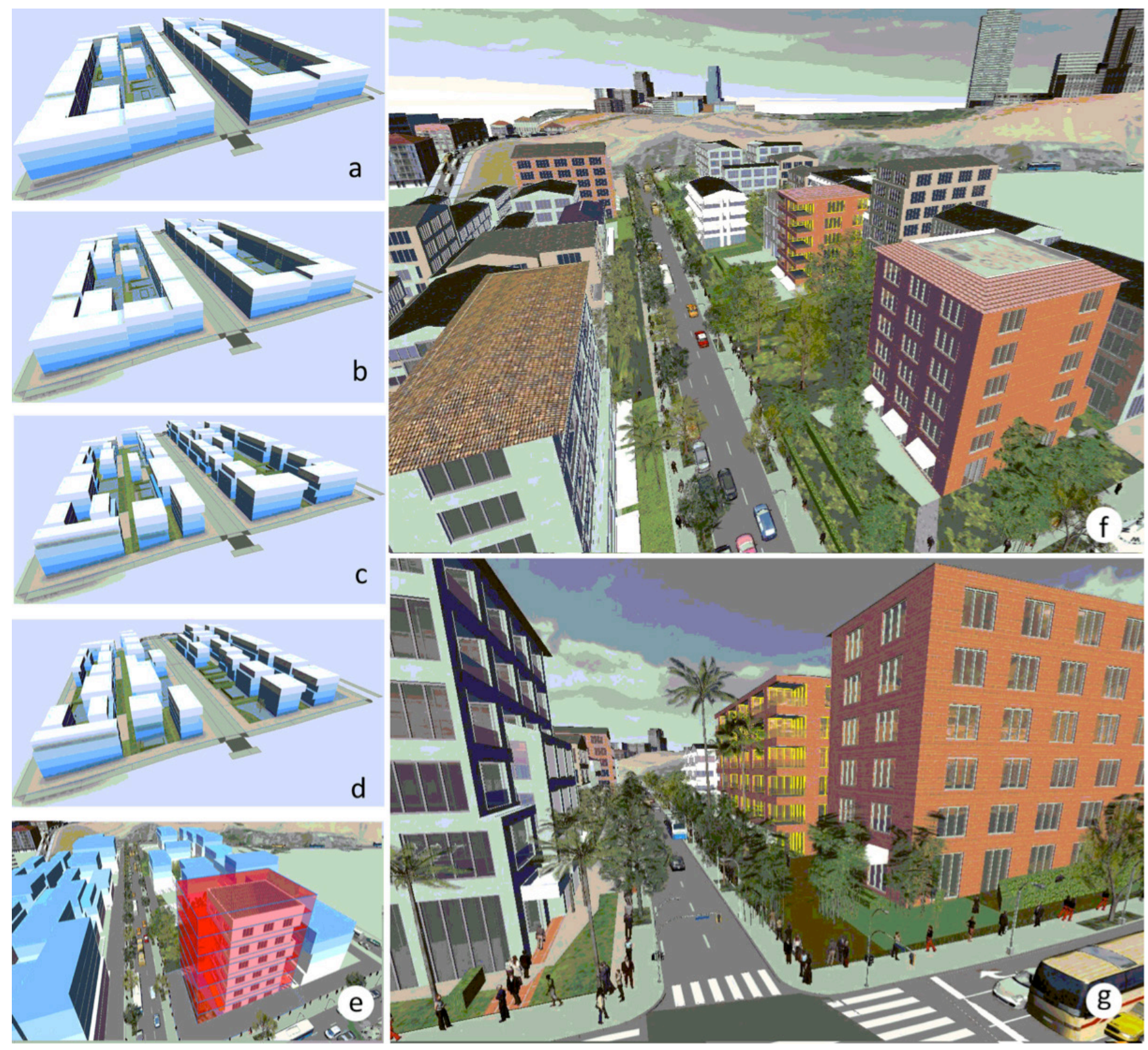

Figura 3: Objetivos da área de psicomotricidade a serem alcançados com a disciplina. Fonte: $O$ autor, 2015. 

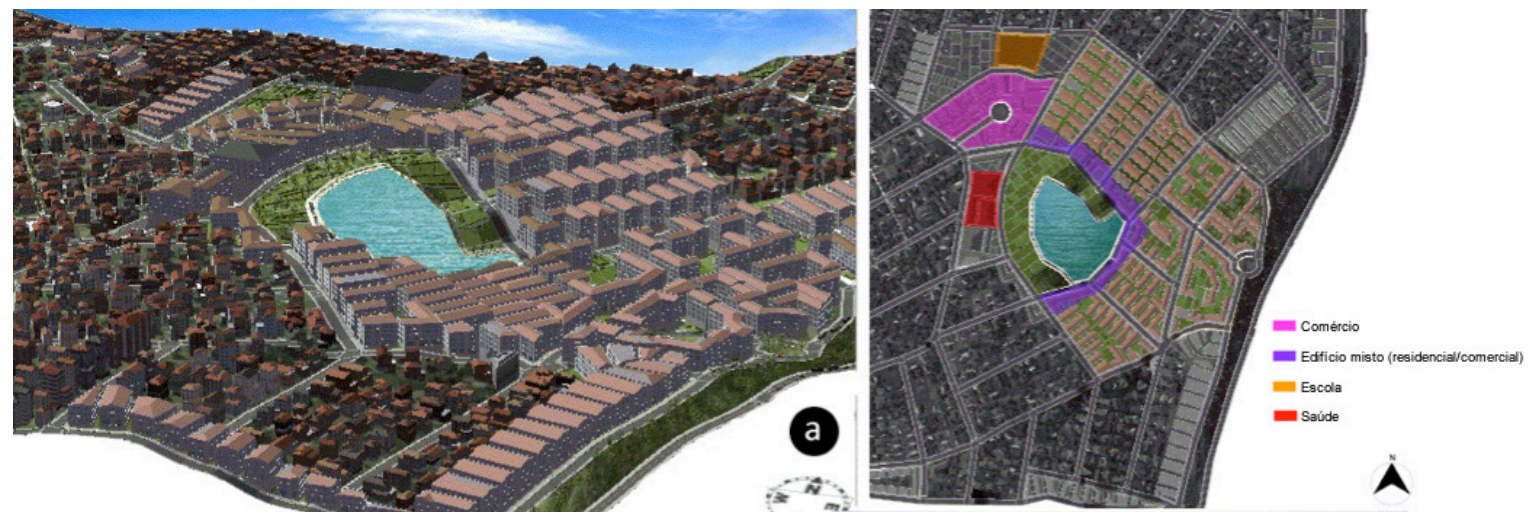

d
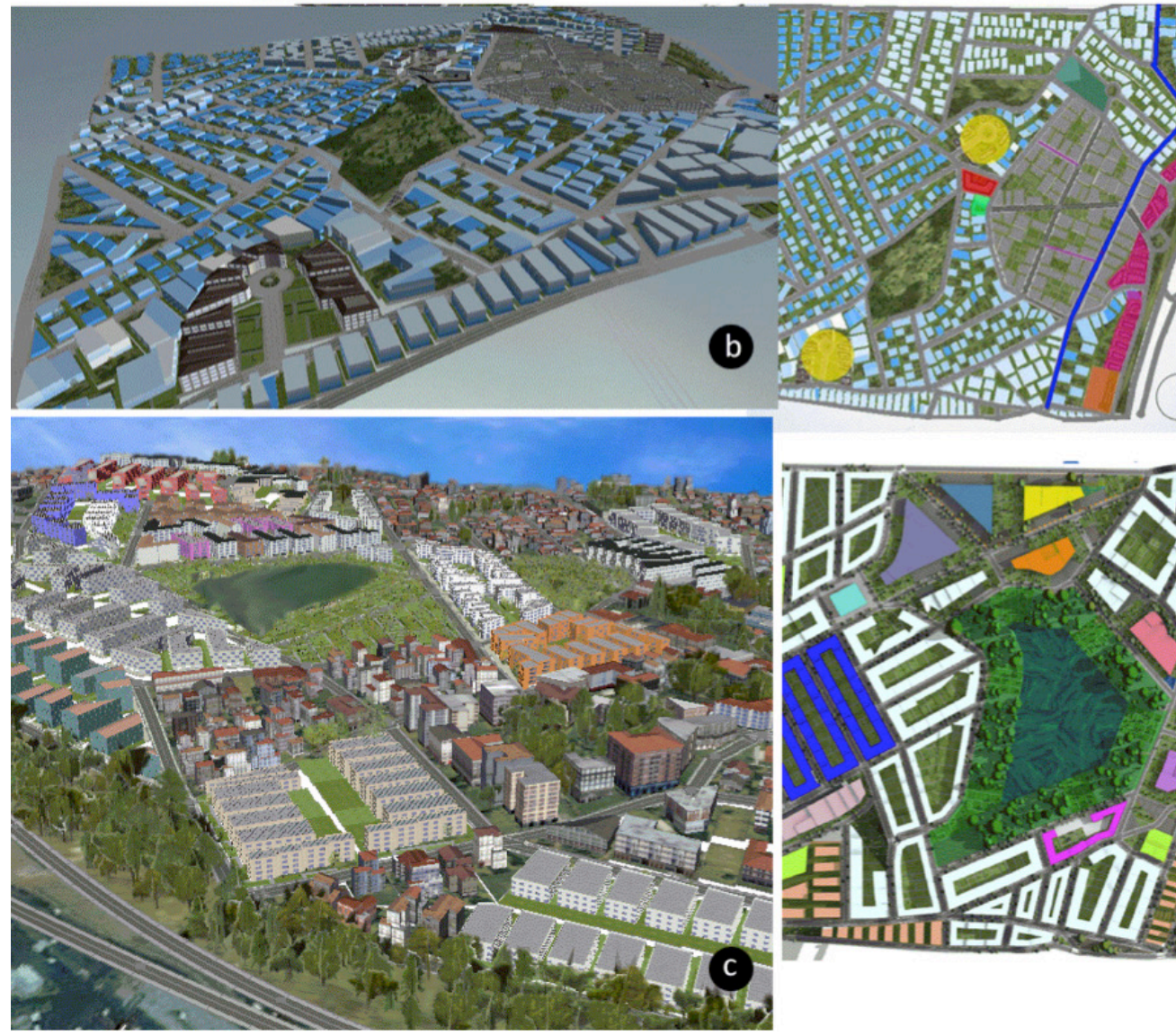

b
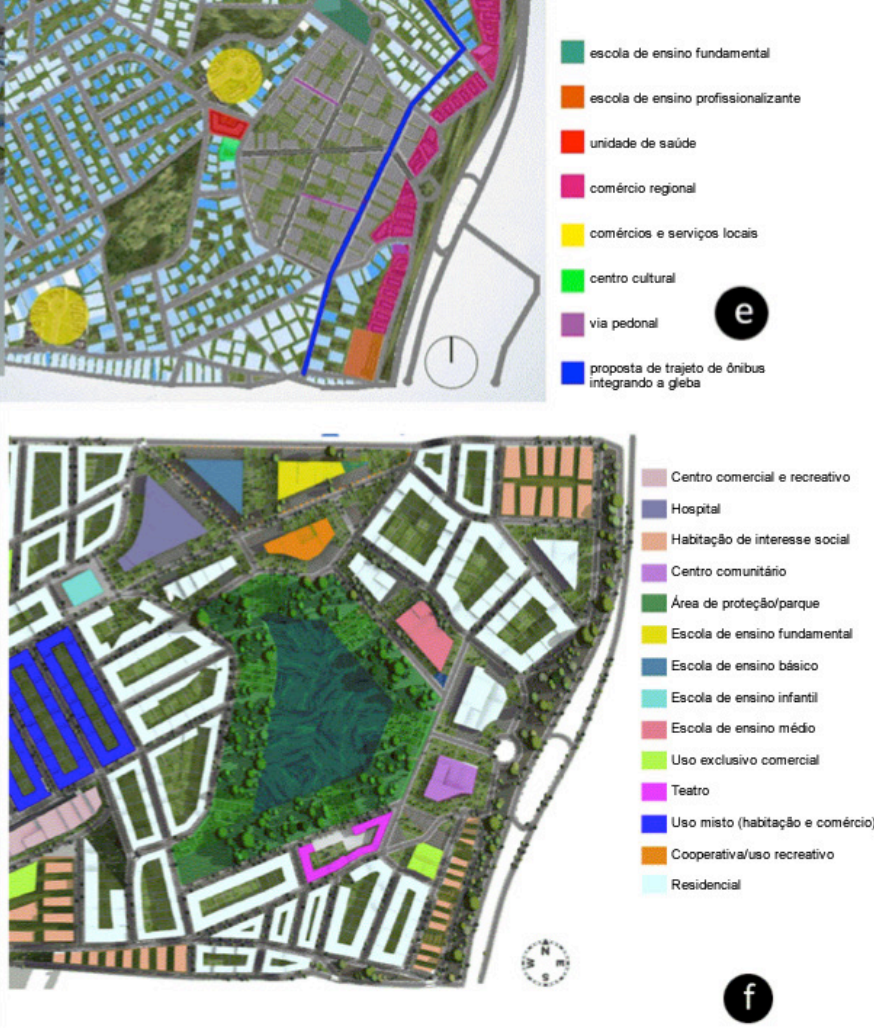

Figura 4: Amostra dos resultados. O software permite a visualização desde perspectivas tiradas de pontos de observadores no interior do terreno, em ruas e praças, e também a visualização esquemática do resultado do assentamento proposto. Fonte: O autor, 2015 


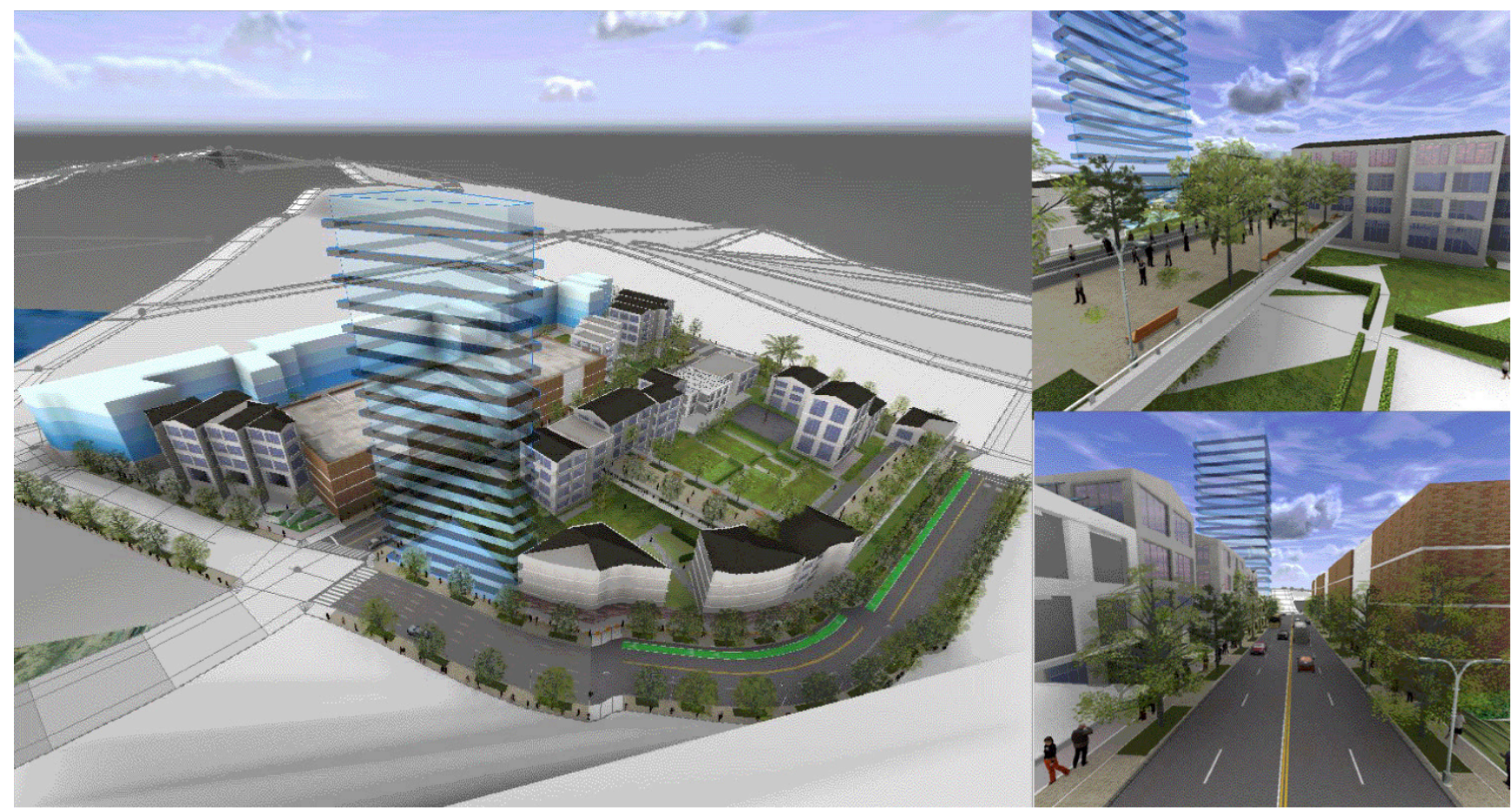

Figura 5: Estudo de uma quadra para inserção de centro comercial com edifício de 15 pavimentos. Fonte: O autor, 2015.
O estudo e as representações de maiores detalhes urbanos e arquitetônicos, entretanto, variaram conforme a capacidade dos computadores pessoais utilizados. Maquetes genéricas como a mostrada na figura $4 \mathrm{~b}$ foram comuns (80\%). Contudo, o software apresenta o recurso de separar áreas a serem detalhadas, como mostra a figura 5, através da qual um estudante detalhou uma quadra para o estudo da implantação de um centro comercial com um edifício de 15 pavimentos, este último representado por um envoltório em transparência. Casos como esse ocorreram com menos de 10\% dos estudantes.

Diante da saturação de tipos semelhantes de soluções apresentadas, validaram-se os trabalhos como objeto de análise e foi considerado que os objetivos educacionais da área volitiva (referentes ao acometimento dos estudantes com a disciplina) foram cumpridos satisfatoriamente $(80 \%)$, que os objetivos gerais foram cumpridos regularmente (70\%), mas que os objetivos da área de psicomotricidade foram insatisfatoriamente cumpridos, como se discute a seguir.

\section{Discussão}

O tempo destinado à disciplina (60 horas/aula) foi considerado o principal fator interferente nos resultados obtidos, pouco responsivos às hipóteses iniciais. O uso dos SIG's contribuiu para a aprendizagem de conceitos básicos do projeto urbano e arquitetônico e ampliou razoavelmente o universo de conhecimentos nos quais os problemas projetuais foram tratados, enquanto permitiu o debate conduzido pelo orientador com o uso da ferramenta em sala. Mas não possibilitou (cerca de 70\%) uma rápida visualização de dados complexos 
9 "Python - Wikipédia, a enciclopédia livre - Wikipedia." <https:// pt.wikipedia.org/wiki/Python>. Acessado em 1 maio 2017. pelos estudantes em situações de estudo e de crítica projetual. Consequentemente, não acelerou a tomada de decisões e não permitiu análises mais abrangentes dos problemas, sendo que as discussões ficaram limitadas a critérios caracterizados pelo conceito inicial adotado por cada estudante, logo após os estudos em Arcgis@ e em Depthmap@. Situações de complexidade foram desta maneira, pontuais e parcialmente discutidas. O uso do SIG visualizado com o Cityengine@ $\odot$ não encaminhou à análise de efeitos de diferentes agentes envolvidos em cenários prospectivos. Ao contrário de criarem hipóteses sobre o desenvolvimento urbano do local, $70 \%$ dos estudantes preferiram utilizar a rapidez da visualização para criar alterações e correções da primeira solução de projeto, detalhando-a realisticamente em aspectos que eram considerados insatisfatórios. Igualmente o fizeram para inserir os projetos arquitetônicos.

Outro importante fator interveniente nos resultados foi o software em si. Enquanto as análises feitas em Arcmap@ e Depthmap@ foram assimiladas com facilidade pelos discentes, quando o professor as executava em sala de aula, o uso do Cityengine@ pelos estudantes, individualmente, inibiu as reflexões relacionadas às consequências da morfologia e zoneamento adotados, no contexto da cidade (cerca de 70\%). A interface do usuário que aquele software apresenta não permitiu, mesmo com as adaptações, o uso de recursos que fossem definidores do zoneamento da área, nem a consequente visualização do uso e ocupação pelo desenho urbano proposto em $50 \%$ dos resultados. Essa utilização das representações esquemáticas (envelopes) pareceu corresponder ao fenômeno da introdução do CAD na arquitetura, há alguns anos, quando computadores eram transformados em pranchetas eletrônicas, com operações mecânicas e repetitivas do desenho a lápis.

Elaborou-se, para pesquisas futuras a hipótese de que é necessário um treinamento anterior para que o estudante possa tirar proveito dos recursos do Cityengine@ no quadro referencial da disciplina. Estimou-se que outro módulo de 60 horas/aula seria razoável como pré-requisito, visando o ensino do software especificamente, mas considerou-se oportuno adotar a estratégia didática de estudar a semântica da morfologia urbana (COUTO, 1969) nos moldes de teorias configuracionais como suporte básico para os conteúdos específicos, como mostra a figura 6 . Acredita-se que isso possa introduzir melhor os propósitos da ferramenta, evitando seu uso em operações mecanizadas que resultam em renderizações realistas de detalhes pouco significativos.

Ainda sobre o software, outro fator preocupante foi testado anteriormente aos estudos de caso. 0 Cityengine $(0)$ utiliza, como se disse, a linguagem $\mathrm{CGA}^{6}$, interface adaptada para o Python ${ }^{9}$. A explicação da arquitetura gerada por esse processo tem por base o uso de regras definidas iterativamente, que vão refinando por repetição as formas simples, criando um maior número de detalhes nas maquetes eletrônicas de modo a eliminar vetores desnecessários. Exemplo disso é a figura 7 exibida no manual do software (ESRI, 2015b). A preocupação foi a de que essa linguagem procedural limitasse a criação e inserção dos projetos básicos, e a representação de contextos urbanos existentes. Entretanto, o manual descreve que o software pode usar geometrias arbitrárias, criadas pelo usuário, denominadas "assets", que incluem desde mobiliários urbanos até edificações inteiras.

Essas geometrias podem ser elaboradas no interior da plataforma ou importadas de outro modelador. Para testar, então, esse recurso, foi elaborado pelo professor, 


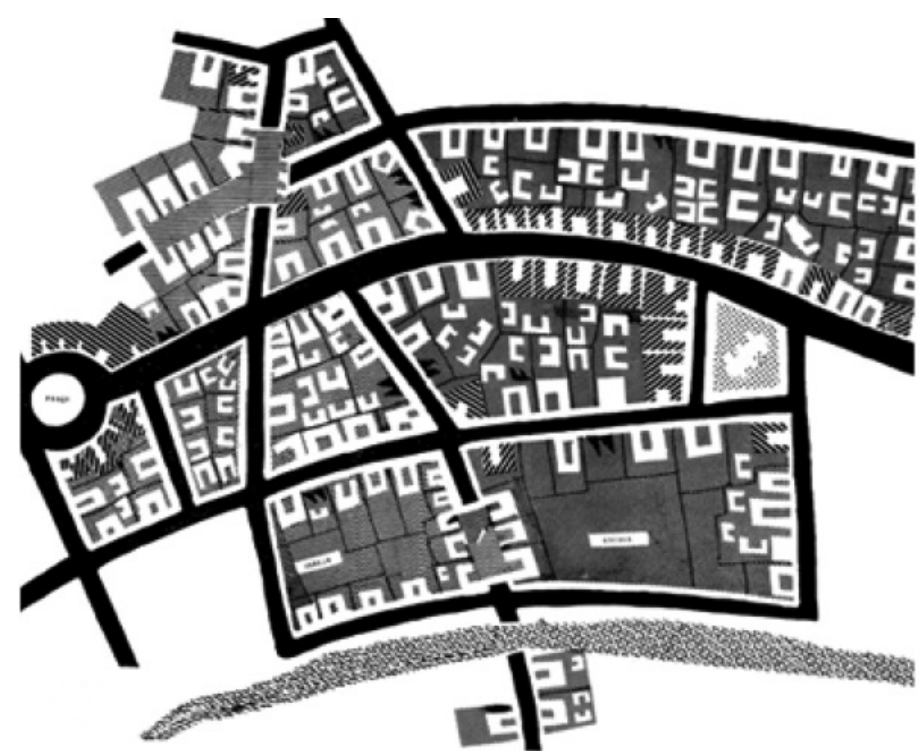

Bairro interiorizado

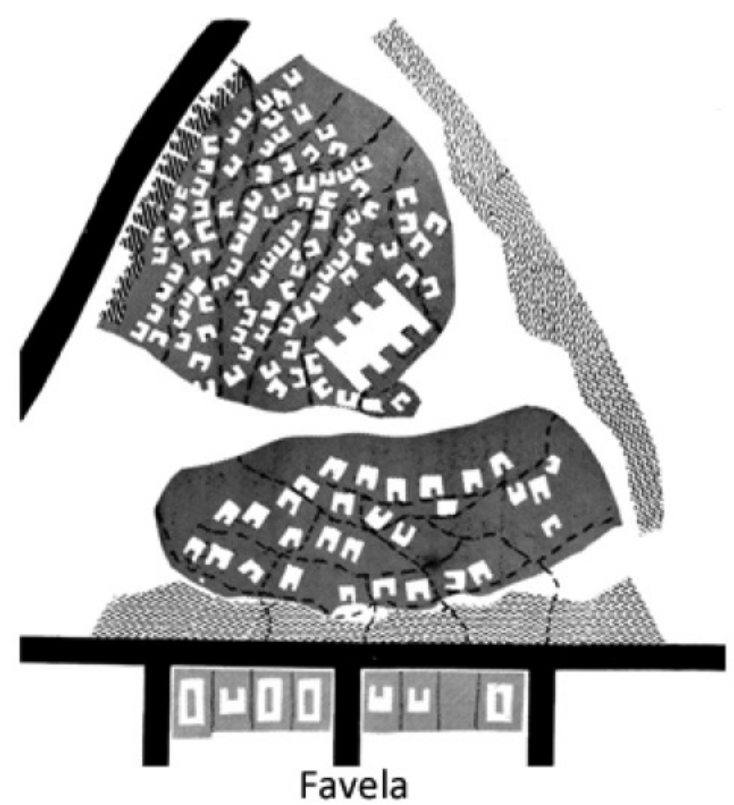

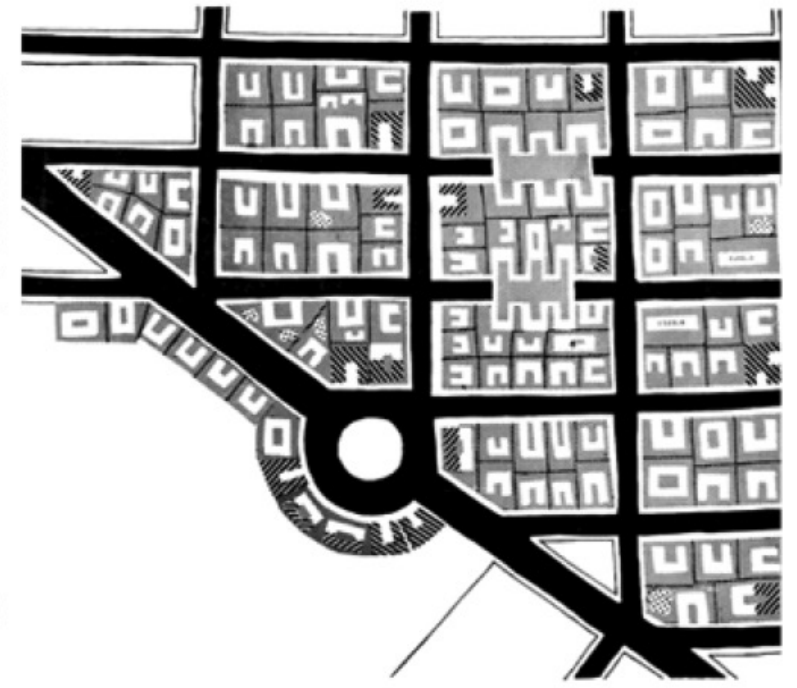

Bairro exteriorizado

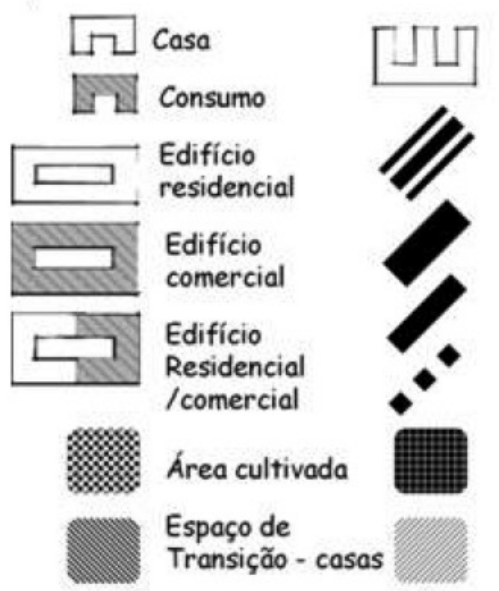

Sociabilidade

Via Primária

Via Secundária

Via Terciária

Via Quaternária

Barreira

Espaço de transição

Figura 6: ॥lustração de estudos da morfologia urbana e suas implicações sócio-espaciais do livro "Um projeto como sistema de relações" (1969). Supõe-se que o estudo dos conceitos configuracionais possa guiar a aprendizagem do Cityengine $\odot$. Fonte: Couto et al (1969). 
Figura 7: Exemplo da linguagem procedural que resulta da gramática da CGA 6. Fonte: Manual do Cityengine $\odot$ (ESRI, 2015b). antes da disciplina, o modelo e visualizações de um aglomerado urbano subnormal em Belo Horizonte, utilizando a textura de fotografias das edificações locais (figura 7). Esse teste mostrou que as representações poderiam evitar repetições características dos desenhos procedurais, gerados pelos pacotes pré-programados de regras contidas no Cityengine $\odot$ que não consideração das características do contexto brasileiro. Foi necessário o conhecimento aprofundado do software para a programação na linguagem CGA6, através da qual o professor compôs pacotes de procedimentos aplicáveis (rules), distribuídos para os estudantes no uso do Cityengine@ 0 . Entretanto, em $80 \%$ dos casos, os projetos arquitetônicos foram inteiramente importados para o interior do Cityengine@), sem o uso das rules adaptadas.

\section{Conclusão}

Alguns cuidados devem ser tomados no uso de SIG's e seus visualizadores para o ensino de Arquitetura e Urbanismo. Além do conhecimento destes pelos professorres, a carga horária deve ser adequada para treinamento dos programas. A escolha de estratégias de ensino deve auxiliar na compreensão dos propósitos do ferramental, afastando o seu uso como simples mecanismo de geração gráfica - a título de uma conclusão geral para este artigo.

Entretanto, o uso de tais recursos em sala, pelo professor, na promoção do debate com a turma, mostrou-se eficiente e garantiu aprendizagem rápida de conceitos que antes - comparando outros semestres - consumiam quase a totalidade do tempo do módulo de 60 horas/aula.

Torna-se evidente que o propósito da facilitação possibilitada pelas ferramentas nas situações em análise não é a simples representação realística de uma solução projetual resultante de análises feitas sobre o problema de projeto no Depthmap@ e no Arcgis $($ - consequentemente, resultando em renderizações de qualidade. Ao contrário, a acuidade e realismo das representações correspondem à ideia de que é possível gerar várias hipóteses e testá-las, uma a uma, com o ferramental, para decidir, então, qual a melhor hipótese projetual a ser adotada.

Mesmo não validando todas as hipóteses iniciais deste estudo, os casos examinados permitiram inferir que tais recursos podem ser utilizados, com a rapidez que proporcionam, para liberar e aumentar o tempo dos estudantes para exercerem a criatividade aonde ela é justamente mais necessária no nosso entender, ou seja, na criação e teste de muitas hipóteses projetuais originais para confrontá-las à complexidade sempre crescente das cidades contemporâneas.

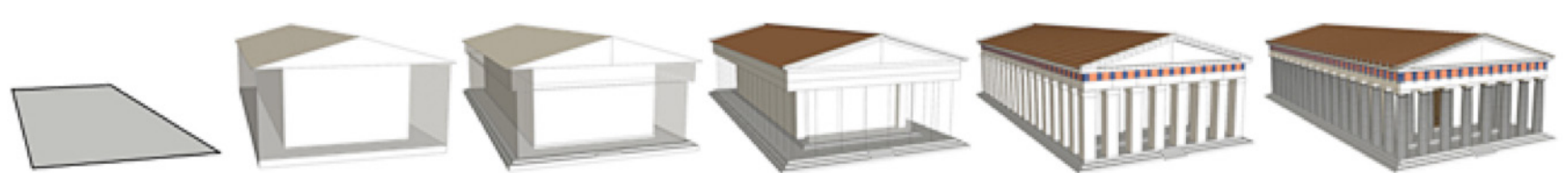


Figura 8: modelo da favela "Vila Dias", no bairro de Santa Tereza, em Belo Horizonte, gerado no Cityengine $\odot$. Com texturas, detalhes arquitetônicos e vegetações identificadas no local, esse modelo excluiu a suspeita da padronização das representações dos modelos por métodos procedurais. Fonte: O autor, 2015.

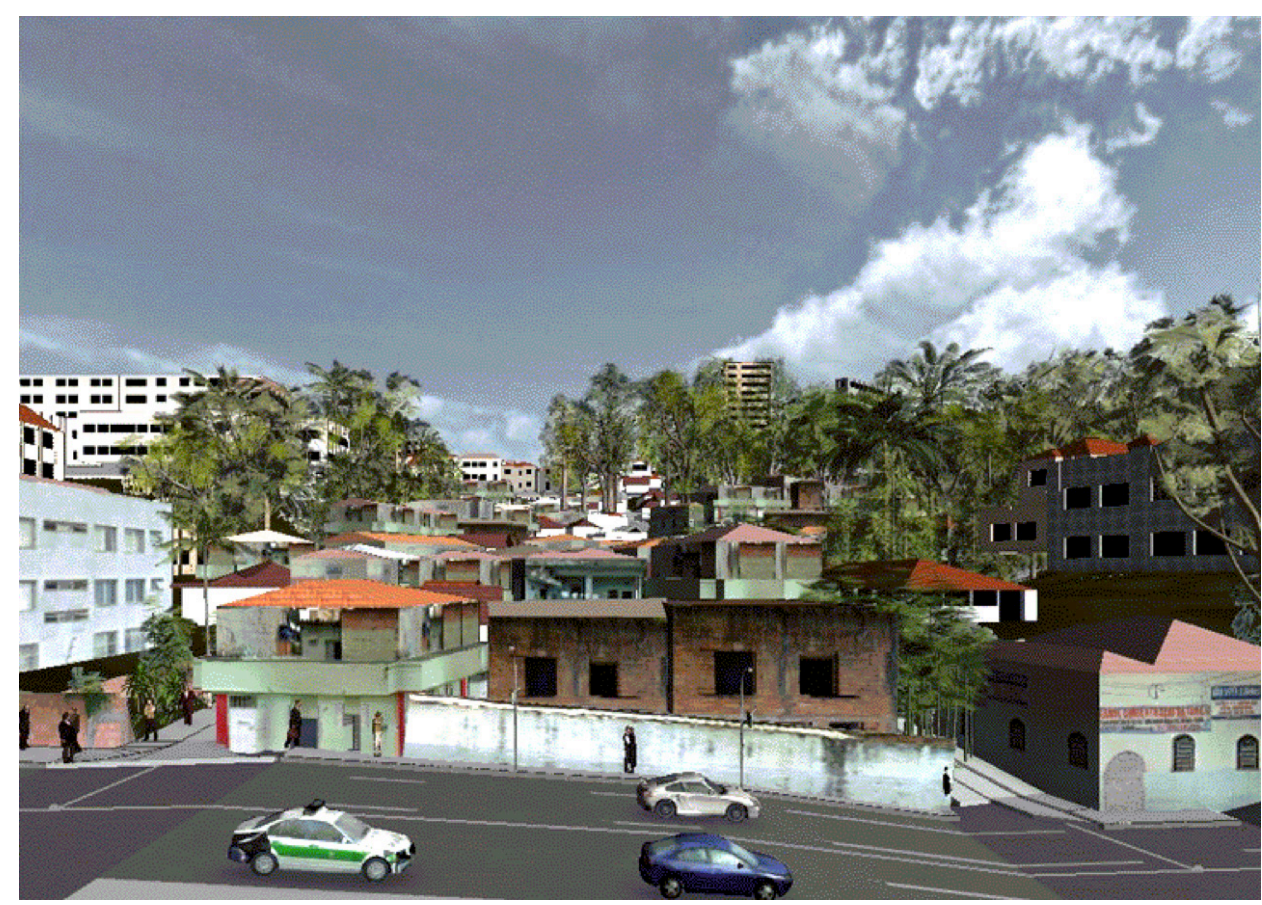

\section{Agradecimento}

Ao CNPq, pelo apoio às pesquisas correlatas que permitiram a realização desses estudos de caso.

\section{Referências bibliográficas}

ANDERSON et al. A taxonomy for learning, teaching, and assessing: A revision of Bloom's Taxonomy of Educational Objectives. New York: Longman, 2001

ARNOLD, M. On the Phenomenology of Technology: the "Janus-faces" of mobile phones. Information and Organization, n. 13, p. 231-256, 2003.

CONRAD, E. Towards Embodied Spatial Interaction. Montreal, Quebec: Topological Media Lab, Concordia University, 2006.

COUTO, B. A. D. ET AL. Um projeto como sistema de relaçoes, uma pre-sintaxe aberta a improvisação social e a significaçoes ainda não constituidas. Belo Horizonte: Escola de Arquitetura da UFMG, 1969. v. 1

DE ALMEIDA, A. T. et al. Multicriteria and Multiobjective Models for Risk, Reliability and Maintenance Decision Analysis. [s.I.] Springer International Publishing, 2015.

ESRI. CityEngine 3D Modeling Software for Urban Environments. Disponível em: <http://www. esri.com/software/cityengine>. Acesso em: 15 out. 2016a.

. Procedural Design. Disponível em: <http://cehelp.esri.com/help/index.jsp?topic=/com. procedural.cityengine.help/html/manual/cga/basics/toc.html>. Acesso em: 1 maio. 2017b.

Arcgis. Disponível em: <https://www.arcgis.com/features/index.html>. Acesso em: 12 out. 2016 
GRAHAN, S. The end of geography or the explosion of place? Conceptualizing space, place and information technology. Progress in Human Geography, v. 22, n. 2, p. 165-185, 1998.

GREENFIELD, A.; SHEPARD, M. Urban Computing and Its Discontents. Disponível em: <http:// situatedtechnologies.net>.

HILLIER, B.; HANSON, J. The social logic of space. Cambridge: Cambridge University Press, 1984.

JORGENSEN, K. A Framework for Geodesign: Changing Geography by Design. [s.I.] Routledge, 2012. v. 7p. 87-87

MONSUR, M.; ISLAM, Z. GIS for Architects: Exploring the Potentials of Incorporating GIS in Architecture Curriculum. ARCC Conference Repository, 2014.

MCCULLOUGH, M. Digital Ground: architecture, pervasive computing and environmental knowing. [s.I.] Massachusetts Institute of Technology - MIT Press, 2004.

TURNER, A. Depthmap. University College of London, UK, 2013. Disponível em: <http://www. spacesyntax.net/software/ucl-depthmap/>

WINOGRAD; FLORES, T. A.; FERNANDO. Understanding Computers and Cognition: A New Foundation for Design. New York: Addison-Wesley Publising Company, 1988. 